\title{
Practical Management of Distance Based Toll System for Urban Expressway
}

\author{
Takamasa Akiyama ${ }^{1}$, Hiroaki Inokuchi ${ }^{1}$ and Masashi Okushima ${ }^{2}$ \\ 1. Department of Civil, Environmental and Applied Systems Engineering, Kansai University, Suita, Osaka 564-8680, Japan; \\ 2. Institute of Technology and Science, Tokushima University, Tokushima 770-8501, Japan
}

\begin{abstract}
According to the extension of ETC (Electronic Toll Collection) System, the distance based toll system has been recently introduced to realize the effective traffic management for urban transport networks. In the study, urban road network in Keihanshin area is analyzed as an empirical study. The traffic assignment technique is modified to estimate the traffic conditions on the network with describing the individual route charging as well as diversion traffic. The impact of implementation of distance based toll onto the real scale transport network can be evaluated to discuss the social benefit of road users. The advanced technique with intelligent information processing can be proposed to determine the optimal combination of parameters in distance based toll function. In the study, the reduction of total travel time of road users is regarded as the index of the social benefit on urban network. Therefore, the estimation model of total travel time is created by neural network without the estimation process for large scale network. After the optimal combination of parameters is determined, practical road pricing policy on the urban network can be analyzed. Finally, the optimal function form of distance based toll is recommended in practical implementation.
\end{abstract}

Key words: Urban expressways, distance based toll, traffic assignment, neural networks.

\section{Introduction}

The proper form of distance based toll has been discussed for basic idea of pricing policy of urban road networks. The traffic assignment as a fundamental technique in the field of traffic engineering can be applied to demonstrate the user equilibrium situation on the road network. In the study, the urban expressway toll system is analyzed to realize the second best toll for urban transport network which consists of urban streets and expressways. According to the extension of ETC (Electronic Toll Collection) System, the distance based toll system has been recently introduced to realize the effective traffic management for urban transport networks [1]. However, the particular type of distance based toll should be determined from the empirical study. Many types of function can be assumed between the distance and the price of toll. Even though the type of toll

\footnotetext{
Corresponding author: Takamasa Akiyama, professor; research field: transport planning. E-mail: akiyama@kansai-u.ac.jp.
}

function is determined, there are some parameters in the function. The modified traffic assignment technique is proposed in the study to evaluate the social benefit in introducing the distance based toll system [2]. The optimization technique with intelligent information processing is proposed as well to specify the parameters in the particular distance based toll function.

The urban road network in Keihanshin area is analyzed as an empirical study. The traffic assignment technique is revised to estimate the traffic conditions on the network with describing the individual route charging as well as diversion traffic in case of distance toll system. The impact of implementation of distance based toll onto the real scale transport network can be evaluated to discuss the benefit of road users. On the other hand, the advanced technique with intelligent information processing can be proposed to determine the optimal combination of parameters in distance based toll function. In the study, reduction of total travel time of road users is regarded as the index of 
the social benefit on urban network. Therefore, the estimation model of total travel time can be created by neural network without traffic assignment process with large scale network.

After the optimal combination of parameters for the distance based toll function is determined, practical road pricing policy on the urban network can be analyzed. Therefore, the toll system of urban expressway is investigated in terms of empirical study. The optimal form of distance based toll system of urban expressway is confirmed with travel time reduction. Finally, the optimal function form of distance based toll is recommended in practical implementation.

\section{The Network Analysis for Urban Expressway}

\subsection{The Distance Based Toll of Urban Expressways}

The urban transport network consists of urban expressways and urban streets. The urban network of Keihanshin area is illustrated in Fig. 1.

The toll road system has been introduced in Japanese urban expressway. As four different zones are shown in the expressway, the distance based toll is applied for central three zones and the uniform toll is applied only for Kyoto district [3, 4].

The proper form of distance based toll has been discussed for basic idea of pricing policy of urban road networks. The traffic assignment as a fundamental technique can be applied to demonstrate the user equilibrium situation on the urban road network. In the study, the urban expressway toll system is analyzed to realize the second best toll for urban transport network which consists of urban streets and expressways. The uniform toll system had been applied in urban expressways for a long time.

According to the recent traffic network extension and requirement of proper traffic management, the distance based toll system has been introduced as an advanced toll system for urban expressway [2]. The step function form of distance based toll has been applied in Hanshin Expressway since January, 2012 as shown in Fig. 2.

Several different forms of distance based toll can be proposed as shown in Fig. 3. The step function form corresponds to the practical distance based toll in the expressway. In the study, the linear distance based toll

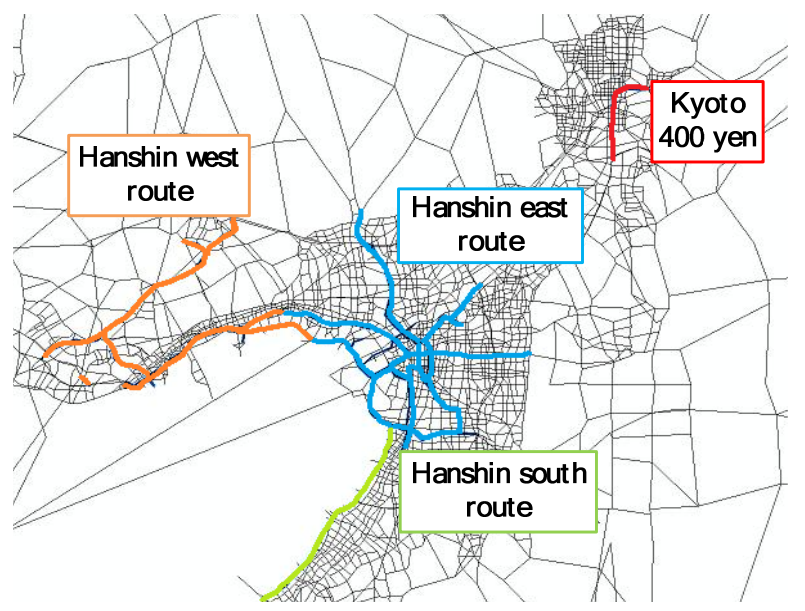

Fig. 1 The urban network of Keihanshin area.

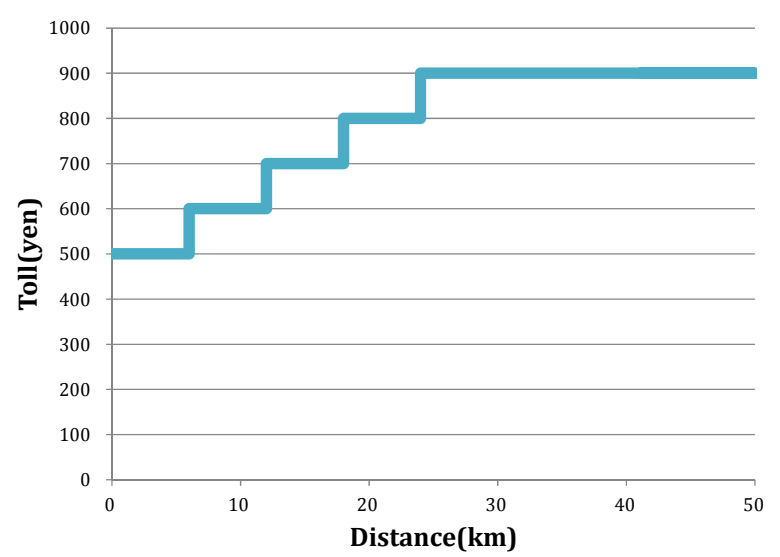

Fig. 2 Urban expressways with distance based toll system.

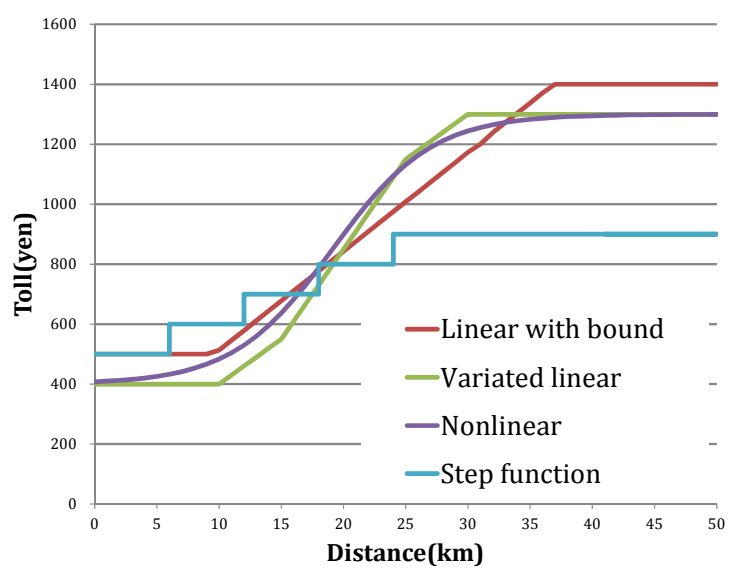

Fig. 3 Several functions of distance based toll. 
is discussed for alternatives of toll system. In particular, the linear function with upper and lower limits should be regarded as a fundamental form. The slope of the linear function indicates the proportion of pricing to the travel distance. Therefore, the linear function can be often applied for basic analysis of function form. The toll with the combination of variable linear functions might be a generalized form to formulate the different proportions of pricing to the travel distance. Furthermore, a monotonically continuous function form of toll can be determined with a few of parameters. Therefore, the nonlinear functional form of toll can be easily handled mathematically to determine the alternative toll systems.

\subsection{Representation of Toll Roads as Urban Expressway}

As mentioned previously, urban transport networks are assumed to consist of streets and urban expressways. The algorithm of user equilibrium traffic assignment can be applied with modification to estimate traffic condition with distance based toll in urban expressway. The impedance of toll between on-ramp and off-ramp for route choice behaviour should be formulated corresponding to the distance based toll system. Different toll should be charged according to the distance between on-ramp and off-ramp. Therefore, representation of toll charge is required in the user equilibrium traffic assignment algorithm [4]. Fig. 4 illustrates the mechanism of route choice in the network with toll roads for urban expressways.

Let us assume the urban expressway route existing between origin " $r$ " and destination "s". The road network between the origin and the destination is divided by three sub networks assuming on-ramp and off-ramp for urban expressway as $(i, j)$. The networks are determined origin to on-ramp for $r-i$, on-ramp to off-ramp for $\mathrm{i}-\mathrm{j}$, and off-ramp to destination for $\mathrm{j}$-s.

The shortest path for each network is determined separately. The value of toll should be taken into account for the generalized travel cost for the expressway path. The value of time is applied to determine the equivalent value of travel time of toll. Therefore, the shortest path for the origin to destination is determined by generalized travel time with combining the shortest paths such as the origin to on-ramp for $r-i$, on-ramp to off-ramp for $\mathrm{i}-\mathrm{j}$, and off-ramp to destination for $\mathrm{j}$-s. According to the algorithm and network representations, the impedance of toll for travel is formulated mathematically to solve the UE (user equilibrium) condition for traffic flow analysis.

\subsection{Representation of Diversion Traffic}

Another importance of traffic assignment technique is to describe the diversion traffic in case of introduction of distance based toll. The travel behaviour of drivers may change under the distance based toll system because the route choice opportunity should be increased comparing to the uniform toll system as previous conditions. The algorithm should be modified to describe the travel behaviour such as origin-street-expressway-street-expressway-street-dest ination.

Therefore, the algorithm should be improved to estimate the diversion traffic on urban expressway. In particular, the modified algorithm is required for shortest path search by OD traffic. The algorithm with four stages is proposed shown in Fig. 5.

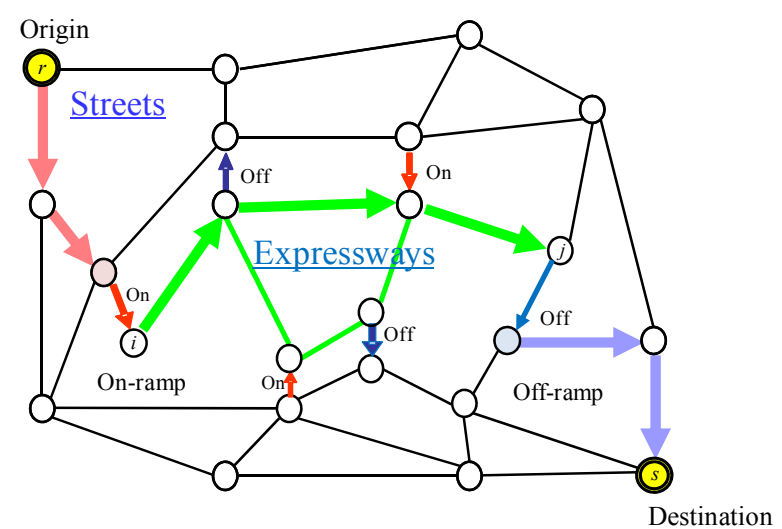

Fig. 4 Representation of urban Networks. 
STEP1 Shortest path between on-off ramps

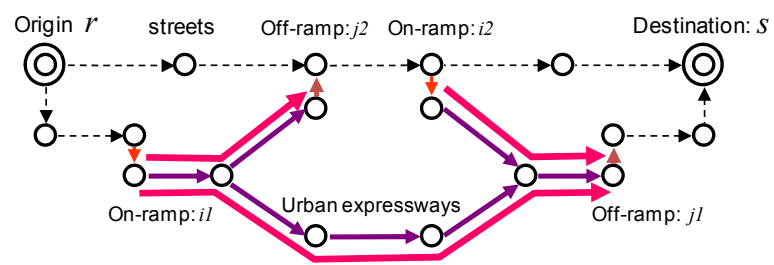

(a)

STEP2 Representation of imaginary links

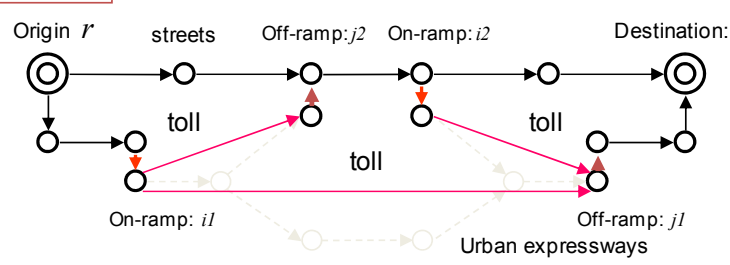

(b)

STEP3 Shortest path in the imaginary network

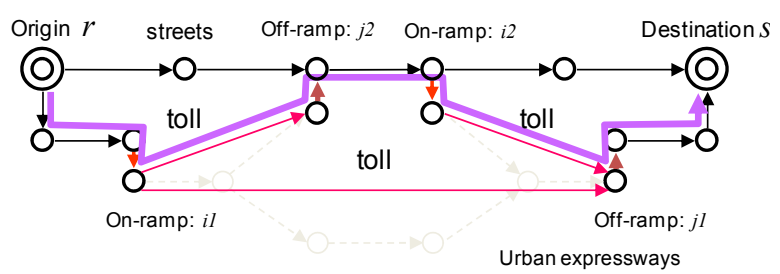

(c)

STEP4 Shortest path between origin and destination

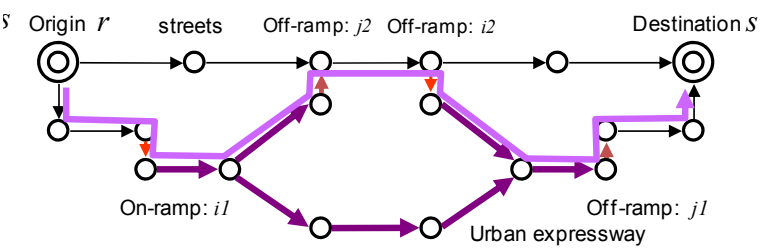

(d)

Fig. 5 Modified algorithm for representation of diversion: (a) Step 1; (b) Step 2; (c) Step 3; (d) Step 4.

The shortest path is searched between the on-ramps and off-ramps in Step 1. The shortest path between the on-ramps and off-ramps can be represented with equivalent value of toll corresponding to the travel distance. The imaginary links are determined as the representation of path travel time and value of toll for the expressway in Step 2. The shortest path is searched on the imaginary network including the representation of the diversion travel in Step 3. After the shortest path is found in the imaginary network, the shortest path on the original network can be determined in Step 4.
This modified algorithm of traffic assignment would describe the behaviour of drivers who may use the expressway sections for two times or more. The imaginary path representation process is required to demonstrate the situations in the algorithm. The modified shortest path algorithm can be introduced into the original algorithm for determination of user equilibrium condition on the network. Therefore, realistic traffic flows on the network can be estimated referring to the diversion traffic under the distance based toll system.

\subsection{The Determination of Distance Based Toll}

The particular form of distance based toll can be determined from the comparison of possible form of toll functions. In the study, the linear function with upper and lower limit can be proposed. The value of toll is counted as 760 yen corresponding to the average distance of travel of expressway as $15 \mathrm{~km}$. The slope of function should be determined as $33 \mathrm{yen} / \mathrm{km}$. Therefore, it is assumed that the function should cross the point as (15 km, 760 yen) with slope of $33 \mathrm{yen} / \mathrm{km}$. As a fundamental case, the upper limit is to be 1,200 yen and the lower limit is to be 400 yen. All installation cases would be determined according to the fundamental case as shown above. The values of upper limit and lower limit are discussed. The level of overall toll is discussed as well. Fig. 6 illustrates the example of installation of toll function. The standard function is shown as $(400,1200,0)=($ lower limit, upper limit, overall level).

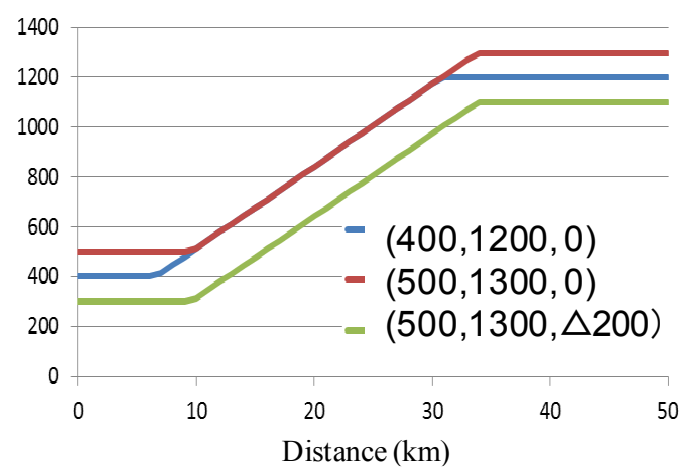

Fig. 6 The installation of distance based toll. 
It is assumed that the lower limit and upper limit are 500 and 1,300, respectively. This corresponds to the function as $(500,1,300,0)$. Furthermore, the overall level of toll would be discussed as well. The function as $(500,1,300,-200)$ is the same form as the function as $(500,1,300,0)$ with 200 yen discount. The bottom linear line corresponds to the function. Therefore, three parameters should be determined to install particular form of toll function.

It is known that lower limit and upper limit of the function as $(500,1,300,-200)$ is equivalent to 300 and 1,100 , respectively. Four values are assumed for lower limit and upper limit, respectively. At the same time, five cases for level of toll are assumed. Therefore, eighty cases $(=4 \times 4 \times 5)$ are considered totally. The results for all cases cannot be required to discuss the proper form of distance based toll. Thirty cases are taken out to investigate the form of distance based toll. The cases with circle are shown in Table 1.

The urban network is represented by 7,826 links and 5,264 nodes. The link flows on urban expressways and urban streets are calculated by traffic assignment techniques as previously mentioned. The revenue of toll and diversion traffic can be estimated from the results of traffic assignment. Therefore, the estimation results for total travel time and the revenue of toll are illustrated in Fig. 7.

The distance based toll with step function is determined as standard form of toll. It corresponds to the present distance based toll in the real world. The value of total travel time for each case is demonstrated in increasing order. The total travel time under the step function toll is indicated in the middle of diagram. The value of total travel time is smaller for 14 cases comparing to the step function toll system. These cases are coloured in Table 1. It can be found that lower level of overall toll may provide the benefit of travel time reduction to the step function toll as traffic demand for urban network is fixed.

On the contrary, the revenue of toll for the expressway can be estimated for each case at the same time. The revenue of toll for each case is shown in decreasing order in Fig. 7.

Table 1 Combination of parameters for distance based toll form.

\begin{tabular}{|c|c|c|c|c|c|c|}
\hline \multirow{2}{*}{$\begin{array}{c}\text { Lower } \\
\text { (yen) }\end{array}$} & \multirow{2}{*}{$\begin{array}{c}\text { Upper } \\
\text { (yen) }\end{array}$} & \multicolumn{5}{|c|}{ The value of discount (yen) } \\
\hline & & $\triangle 200$ & $\triangle 100$ & 0 & 100 & 200 \\
\hline \multirow{4}{*}{300} & 1200 & 0 & & $\Delta$ & & 0 \\
\hline & 1300 & & 0 & & $\boldsymbol{\Delta}$ & \\
\hline & 1400 & & 0 & & 0 & \\
\hline & 1500 & 0 & & $\boldsymbol{\Delta}$ & & 0 \\
\hline \multirow{4}{*}{400} & 1200 & & & 0 & & $\bigcirc$ \\
\hline & 1300 & 0 & $\boldsymbol{\Delta}$ & & O & \\
\hline & 1400 & & 0 & & $\boldsymbol{\Delta}$ & 0 \\
\hline & 1500 & $\boldsymbol{\Delta}$ & & 0 & 0 & \\
\hline \multirow{4}{*}{500} & 1200 & 0 & & 0 & & $\Delta$ \\
\hline & 1300 & & 0 & & 0 & \\
\hline & 1400 & 0 & & $\Delta$ & & $\bigcirc$ \\
\hline & 1500 & & 0 & 0 & & $\Delta$ \\
\hline \multirow{4}{*}{600} & 1200 & 0 & & $\Delta$ & & 0 \\
\hline & 1300 & & $\Delta$ & & 0 & \\
\hline & 1400 & & 0 & & 0 & \\
\hline & 1500 & 0 & & & $\boldsymbol{\Delta}$ & 0 \\
\hline
\end{tabular}

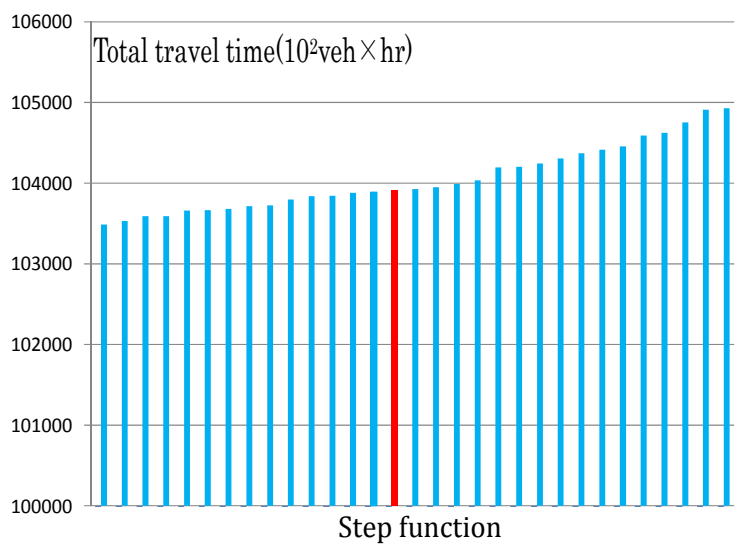

(a)

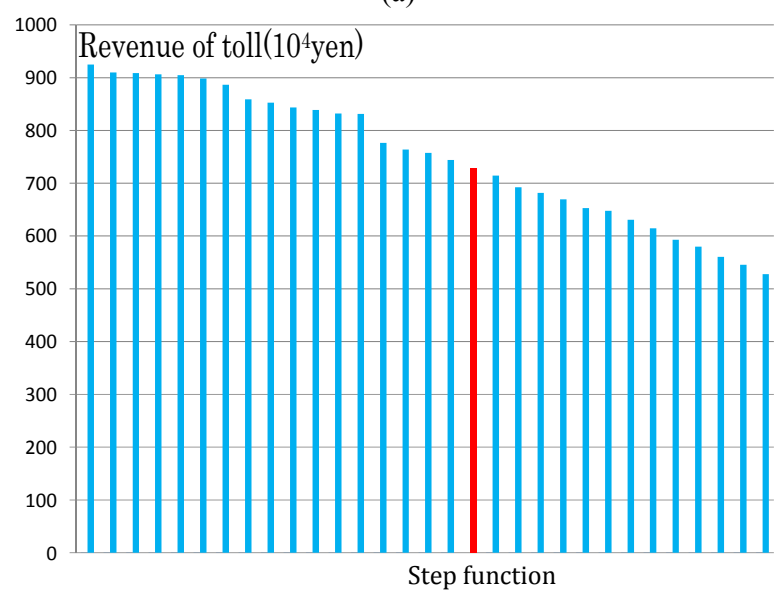

(b)

Fig. 7 Estimation results of evaluation values: (a) total travel time; (b) revenue of toll. 
The case of step function toll is shown in the middle of the diagram. The larger values of toll revenue are observed in 17 cases to the step function toll. This reflects on the increase of traffic volume on urban network. It is known that the introduction of proper distance based toll system may increase revenue of toll.

The comparison of different forms of distance based toll has been discussed with small number of parameter combination. The evaluation results for even small number of cases may provide the significant comparison of different forms of distance based toll.

\section{Evaluation with Neural Network Model}

\subsection{Neural Network Model}

The intelligent information technique is applied to estimate the evaluation values for the different forms of distance based toll. A large number of parameter combinations are assumed such as the lower limit, the upper limit and the discount level of toll even in the linear function. Theoretically, it is possible to determine the optimal form of distance based toll among many alternative combinations of parameters. The MPEC (mathematical problem with equilibrium constraints) problem can be formulated that the user benefit of reduction of travel time on urban network is maximized subject to user equilibrium condition [5]. The problem can be formulated as follows:

$$
\min Z_{S}=\sum_{a \in A} x_{a} \cdot t_{a}(w)
$$

s.t.

$$
\left\{\begin{array}{l}
\min Z_{U}=\sum_{a \in A} \int_{0}^{x_{a}} t_{a}(w) d w+\sum_{i j \in \Omega_{h}} \frac{p_{i j}}{\gamma} f_{(i, j)}^{r s} \\
\text { s.t. } \\
\sum_{k \in K_{r s}} f_{k}^{r s}-Q_{r s}=0, \quad \forall r s \in \Omega \\
x_{a}=\sum_{k \in K_{r s}} \sum_{r s \in \Omega} \delta_{a, k}^{r s} f_{k}^{r s}, \quad \forall a \in A \\
f_{k}^{r s} \geq 0, \quad x_{a} \geq 0
\end{array}\right.
$$

where, $x_{a}$ is traffic flow on link $a, f_{k}^{r s}$ is traffic flow on path $k$ for OD pair $r-s, t_{a}\left(x_{a}\right)$ is the performance function for link $a, Q_{r s}$ is traffic demand for OD pair $r-s, p_{i j}$ is the price of toll for ramp pair $i-j$, and $\gamma$ is the value of time. The objective function is formulated as minimizing the total travel cost for urban networks. The constraint should be formulated as user equilibrium condition with referring to the price of toll for travel on the urban expressway.

As the large time consuming effort is required in the traffic assignment for large scale network, it seems to be difficult to apply the traffic assignment technique to realize the user equilibrium condition for every evaluation times in the previous formulation. Therefore, the practical optimization approach would be required.

In the study, the approximation of evaluation values would be calculated without the traffic assignment process. The intelligent information technique can be applied to create the estimation model of approximation value of traffic condition.

The estimation model is created according to the procedure [6]: (1) The form of distance based toll is determined with parameters of linear function; (2) Traffic flows on the urban network are estimated with traffic assignment techniques; (3) The indices of evaluation values are calculated from the estimation results of traffic flow. Neural network $(\mathrm{NN})$ model is proposed to develop the approximation model.

In the NN model, the input layer corresponds to the function form of distance base tolls. Once the connecting weights of NN are determined, the total travel time and revenue of toll can be estimated following the information processing from the input layer. Since the estimation process is proposed to omit traffic assignment procedure with large scale networks, many evaluation results for combination of parameters in distance based toll can be calculated rather instantly.

The evolutional optimization approaches such as genetic algorithm can be applicable for the similar problem of total travel time minimization with combinations of parameters. However, the practical 
optimization is not essential for reduction of calculation time in this formulation because the omission of calculation in traffic assignment corresponding the problem from Eq. (2) to Eq. (5) should be the most effective.

The neural network can describe the complex interaction between the independent variables and the objective variables. As the upper limit, the lower limit and the discount level are determined, the total travel time and revenue of toll can be estimated approximately. The structure of neural network for approximation model of evaluation values can be shown in Fig. 8.

The neural network consists of 3-3-2 neurons for each layer. The estimation results for 30 cases in previous section are applied as training data for parameter determination of neural network. According to the formulation of the problem in Eqs. (1) to (5), it is known that the total travel time can be counted by vehicles hour and the revenue of toll. Both values for evaluation can be determined from the network traffic flows on the urban networks as results of UE traffic assignment in Eqs. (2) to (5). In the estimation, the process of back propagation for training is iterated at maximum 10,000 times. It is basic estimation procedure to determine the connecting weights between neurons.

The approximation model for the evaluation value is created with neural network representation. The total travel time and revenue of toll can be estimated approximately without traffic assignment process. The relation between the approximation of neural network and the estimation from traffic assignment can be illustrated. The relation for total travel time is illustrated in Fig. 9a. The RMSE (root mean square error) for total travel time is counted as 9,981 (veh·hour).

The approximation value of total travel time by NN is quite similar to the value from the traffic flow estimation by UE traffic assignment. On the other hands, the revenue of toll is estimated as well simultaneously by NN model. Therefore, the relation between the estimation of traffic assignment and approximation of $\mathrm{NN}$ is shown in Fig. 10b. The RMSE is counted as $931 \times 104$ yen.

According to both estimation results, the strong correlation can be observed from the figure between estimation of $\mathrm{NN}$ model and results of traffic flow analysis. Therefore, the approximation of $\mathrm{NN}$ model

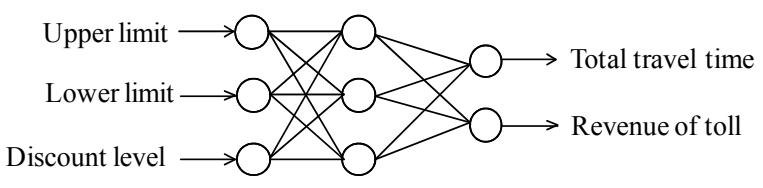

Fig. 8 Representation of neural network model

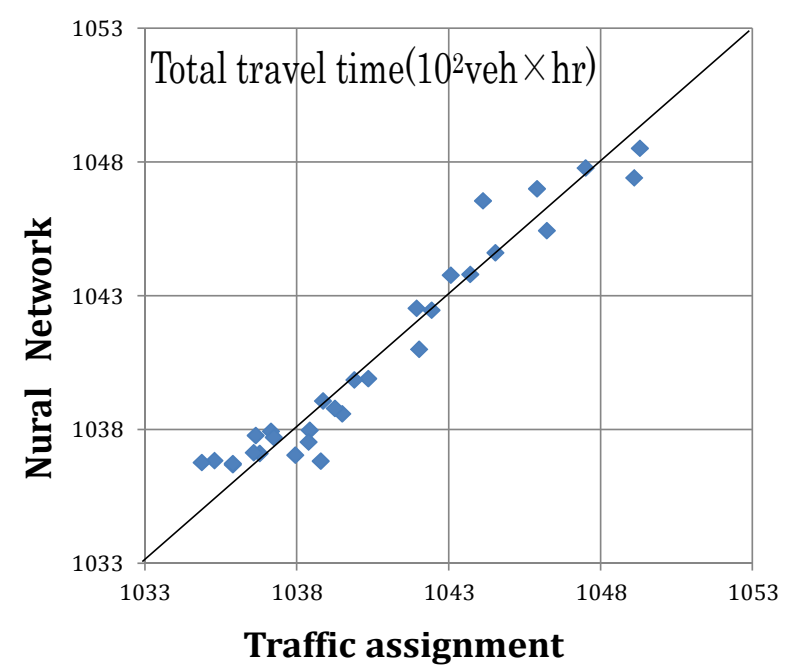

(a)

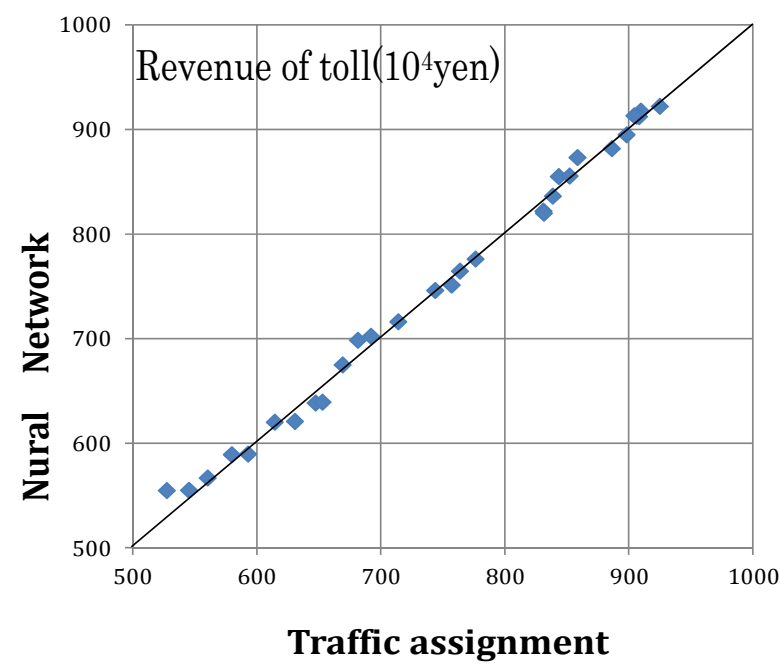

(b)

Fig. 9 The estimation results of neural network for training data: (a) total travel time; (b) revenue of toll. 
can be applicable to the practical evaluation. In terms of the revenue of toll for another evaluation value, the similar result can be confirmed with small error. Therefore, the estimation of NN model is practically applied in the evaluation of distance based toll installation without the estimation of traffic assignment procedures.

\subsection{The Installation of Proposed Distance Based Toll}

The traffic flow on the network with several combinations of parameters is estimated by traffic assignment for validation of the NN model. The 12 cases are indicated with black triangle as $\boldsymbol{\Delta}$ in Table 1 . The total travel time and the revenue of toll are estimated from the NN model without the evaluation from the traffic assignment estimation. The relation between the $\mathrm{NN}$ estimation and traffic assignment results is illustrated in Fig. 10 for validation of the NN model.

The strong correlation can be observed even for the validation data in the figure. It is confirmed as well that the NN model with small number of training data sufficiently estimates the evaluation indices obtained through the process of the traffic assignment.

The optimal form of linear distance based toll would be determined according to approximation of the evaluation values. The user benefit of total travel time reduction for urban road networks is applied as a measure of effective form of distance based toll. The parameters of distance based toll such as lower limit, upper limit and discount level are determined by 10 yen as unit cost. The lower limit is varied between 300 yen to 600 yen for 31 cases. The upper limit is varied between 900 yen to 1,500 yen for 61 cases. Similarly, the discount level is varied between -200 and 200 for 41 cases. Therefore, the number of combination for parameters is counted as 77,531 $(=61$ $\times 31 \times 41)$. It seems to be difficult to estimate the traffic flow conditions for urban network with traffic assignment techniques because of large number of combination. The approximation model with neural network is applied to estimate the evaluation values as total travel time and revenue of toll.

According to the estimation of total travel time for all cases from the NN model, the optimal case is pointed out with the minimum value of total travel time. At the same time, the revenue of toll is estimated

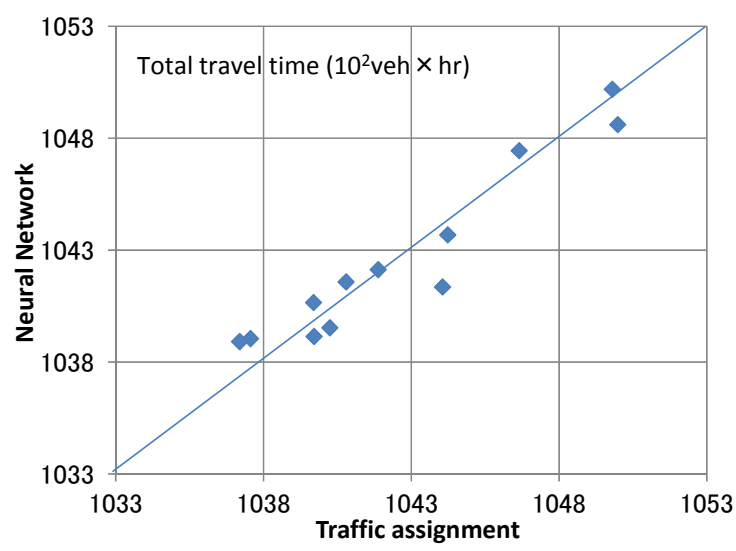

(a)

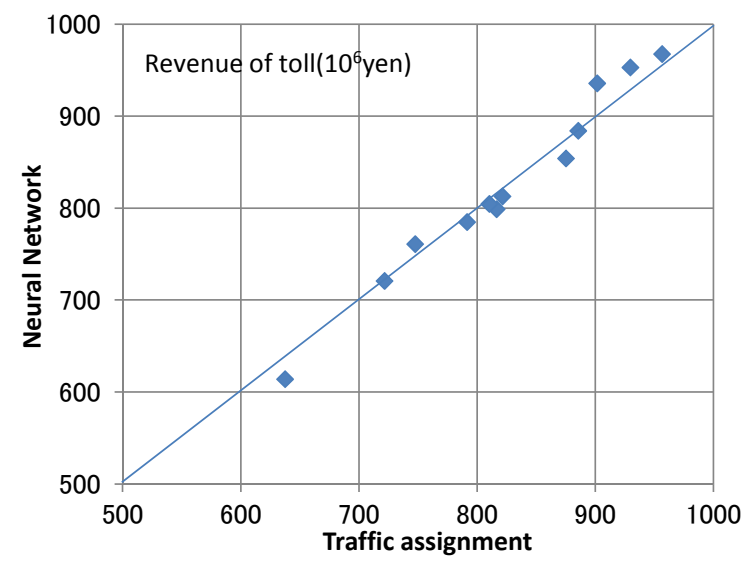

(b)

Fig. 10 The estimation results of neural network for validation data: (a) total travel time; (b) revenue of toll.

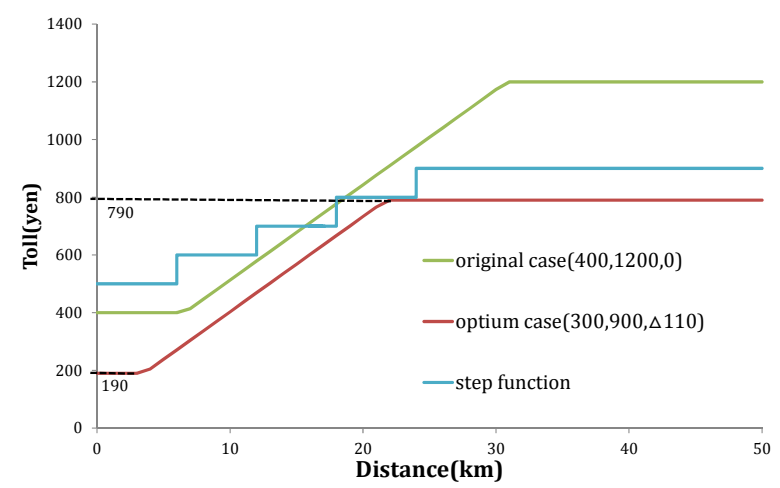

Fig. 11 The optimal form of distance based toll. 
for the case. The optimal case can be indicated as (lower limit, upper limit, discount level $)=(300,900$, $-110)$. The form of the function is illustrated with the original case as $(400,1,200,0)$ and step function form as current. As the discount level is equal to 110 yen, the real lower limit and upper limit are determined as 190 yen and 790 yen respectively. The overall form of the distance based toll is similar to the original installation as $(400,1,200,0)$. The smaller lower limit is recommended according to the optimization of the combination of parameters.

The proposed approximation model by neural network seems to be profitable to find the optimal case among the large number of alternative cases. The optimal distance based toll is recommended with the discount level of 110 yen to the linear function with lower and upper limit as $(300,900)$ for the distance based toll. This effective modification of toll function can be regarded as an advantage of distance toll system. Actually, the NN model can be applied not only for the linear function toll but also for the nonlinear function with several determined parameters with the same manner.

The traffic flow on the network with the optimal distance based toll as $(300,900,-110)$ can be estimated through the traffic assignment previously mentioned. Table 2 summarizes the essential evaluation values from the traffic flow estimation.
The total travel time for urban expressways is increased by proposed distance based toll comparing to the step function toll. On the contrary, the total travel time for urban network involving the ordinal streets is reduced. It causes inflow traffic increase for urban expressway with lower level of toll charge. The average travel distance decreases as well. It can be concluded that the distance based toll may provide the user benefit with optimal form determination.

The requirements should be mentioned to determine the proper form of the distance based toll. The linear distance based toll should be recommended mainly because of improvement of traffic efficiency on urban network. Therefore, total travel time for urban network should be reduced.

On the other hand, the daily refund cost for construction of urban expressway should be required in the definition of toll. The requirement of revenue is determined as about 540,000 thousand yen from long term traffic estimation.

In terms of the distance based toll, the user benefit can be estimated by the reduction of total travel time for overall urban networks. The magnitude of user benefit can be evaluated comparing to the case of no toll charge for the network. The statistics from the traffic flow estimation with no toll cases is shown in the table. The total travel time for the overall network is slightly increased in the step function as well as in

Table 2 Evaluation of the optimal distance based toll.

\begin{tabular}{|l|l|l|l|l|l|l|l|l|}
\hline \multirow{2}{*}{ Toll } & \multicolumn{9}{|c|}{ Urban expressways } & \multicolumn{3}{c|}{ Urban networks } \\
\cline { 2 - 9 } & $\begin{array}{l}\text { Revenue } \\
\left(10^{4} \text { yen }\right)\end{array}$ & $\begin{array}{l}\text { Vehicles } \\
(\mathrm{vehs})\end{array}$ & $\begin{array}{l}\text { Average } \\
(\mathrm{yen} / \mathrm{veh})\end{array}$ & $\begin{array}{l}\text { Average } \\
(\mathrm{km})\end{array}$ & $\begin{array}{l}\text { Diversion } \\
(\mathrm{vehs})\end{array}$ & $\begin{array}{l}\text { Travel time } \\
(\mathrm{veh} \times \mathrm{hr})\end{array}$ & $\begin{array}{l}\text { Travel time } \\
(\mathrm{veh} \times \mathrm{hr})\end{array}$ & $\begin{array}{l}\text { User benefit } \\
\left(10^{4} \text { yen }\right)\end{array}$ \\
\hline $\begin{array}{l}\text { Step } \\
\text { function }\end{array}$ & 72,797 & $1,052,124$ & 692 & 16.2 & 22,609 & 364,841 & $10,390,510$ & - \\
\hline $\begin{array}{l}\text { Original } \\
\text { case }\end{array}$ & 74,402 & $1,127,500$ & 660 & 14.7 & 29,818 & 350,044 & $10,384,260$ & 2,625 \\
\hline $\begin{array}{l}\text { Optimum } \\
\text { case }\end{array}$ & 58,626 & $1,245,661$ & 471 & 139. & 42,877 & 372,121 & $10,360,610$ & 12,558 \\
\hline $\begin{array}{l}\text { Toll free } \\
\text { case }\end{array}$ & 0 & $1,699,066$ & 0 & 11.8 & 218,437 & 468,121 & $10,381,880$ & - \\
\hline
\end{tabular}




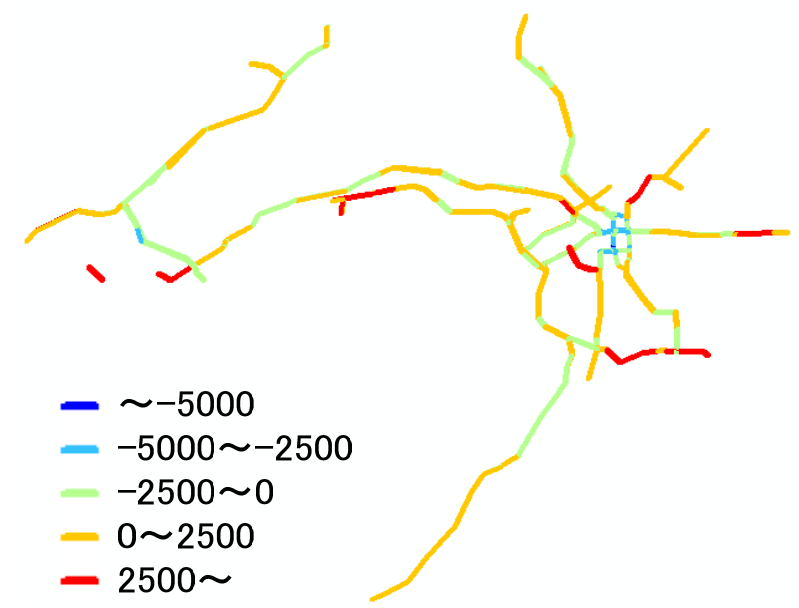

Fig. 12 The traffic flow change on the proposed distance based toll (unit in veh.).

the original function. The total travel time is decreased in the optimum function case. The fact shows the advantages of optimum distance based function as well. It is assumed that the OD traffic demand is not varied in the estimation of the study. Therefore, the larger travel time reduction might be observed with the variable OD traffic demand as a more realistic assumption for the urban networks.

Traffic flow change between linear function toll and step function on the network can be estimated as well. Fig. 12 illustrates the link traffic flow change on the Hanshin expressway.

As the lower price of toll is introduced comparing to the step function toll, traffic flow is reduced on the loop road in the city centre. On the contrary, traffic flow in the surroundings should be increased.

\section{Concluding Remarks}

The practical distance based toll system for urban expressway is discussed. In particular, the proper form of toll can be determined among many alternatives. The main findings are summarized as follows:

(1) Many values of toll for travel between on-off ramps can be determined in distance based toll system. It is realized that the proper form of distance based toll can be determined in terms of the efficiency of urban traffic. It can be summarized that the optimization problem of the distance based toll with different forms can be formulated as MPEC according to user benefit of travel time reduction;

(2) Even though the user equilibrium analysis is effective to estimate the traffic flows on urban network, the effort of estimation must be too much to evaluate all alternative cases concerning with the distance based toll system. The approximation model to estimate the evaluation values is proposed with neural network representation. The evaluation values such as total travel time and revenue of toll can be determined from the NN model with significant level of estimation;

(3) Since the evaluation of feasible alternatives of the distance based toll can be estimated by the $\mathrm{NN}$ model rather instantly, the optimal form of the distance based toll is easily determined among many alternatives. In particular, it is useful to determine the several parameters in the distance based toll. The approximation model with $\mathrm{NN}$ is easily applicable in the practical problem. Finally, the optimal linear function is derived to produce the effective traffic flow on the urban expressways.

The approximation of evaluation values for distance based toll can be applied to the large scale expressway networks. For further study, the interaction between user benefit as reduction of travel time and the revenue of toll for management sector of urban expressway should be discussed for the comprehensive traffic management with pricing policy on urban expressways. Furthermore, the nonlinear function tolls with several parameters would be discussed as well for the proper distance based toll determination.

\section{Acknowledgments}

The authors would like to give the warmest thanks to Hanshin Expressway Company Limited for gathering the traffic data and to Ms. Rei Asahara for helping the calculation of traffic assignment for evaluations. 


\section{References}

[1] Akiyama, T. 2008. "Advanced Traffic Management of Urban Expressway Combining with Pricing Policy." Expressways and Automobiles 51 (12): 5-8.

[2] Mun, S., Akiyama, T., and Okushima, M. 2007. "Second-Best Congestion Pricing in Road Network: Cordon Pricing and Existing Toll-Roads." Journal of Applied Regional Science 12: 15-25.

[3] Akiyama, T., and Okushima, M. 2004. "Pricing Policy of Urban Expressway Using User Equilibrium Analysis." Annual Report on Transportation Economics 47: 169-78.

[4] Okushima, M., and Akiyama, T. 2006. "Discussion on
Distance-Based Toll System for Urban Expressway with User Equilibrium Traffic Flow Analysis.” Annual Report on Transportation Economics 49: 81-90.

[5] Akiyama, T., Okushima, M., and Inokuchi, H. $2011 \mathrm{a}$. "Empirical Implementation of Distance based Toll for Urban Expressway." In Proceedings of the 1st Conference of Transportation Research Group of India, 1-12.

[6] Akiyama, T., Inokuchi, H., and Okushima, M. $2011 \mathrm{~b}$. "The Installation of Fare System on Urban Expressway Considering Diversion Traffic." Journals of the Japan Society of Civil Engineers 67 (5): 1225-32. 